\title{
PREMATURE OBLITERATION OF THE FORAMEN OVALE
}

\author{
BY \\ G. AUSTIN GRESHAM \\ From the Department of Pathology, University of Cambridge
}

Received August 16, 1955

Obliteration of the foramen ovale occurring during intrauterine life is a rare condition. It throws some light on the mechanism of development of endocardial fibroelastosis and also upon the factors concerned in determining the size of the aorta and of the cardiac chambers.

\section{CASE History}

The patient was the second child of a mother (aet. 21) whose previous obstetric history was normal. Apart from two periods of rather rapid gain of weight for which no cause could be found, the pregnancy was uneventful. The child was eleven days postmature; labour was induced with an enema and lasted forty-five minutes.

The infant cried lustily but was cyanosed: the heart was clinically normal. Abnormalities were present in all four limbs. The left radius and ulna were absent and rudimentary digits were present on the skin over the distal end of the limb. Terminal phalanges were absent in the fingers of the right hand, and four toes were present on each foot with a rudimentary fifth digit on the left foot.

Cyanosis and dyspnœa became more intense and the child died three hours after birth despite the use of oxygen.

\section{NECROPSY}

The body was that of a full-term male infant (weight $3430 \mathrm{~g}$.). The limbs were abnormal as previously described. The lips were blue-black in colour.

On the septal wall of the right atrium a hemispherical grey-white area $(10 \times 12 \times 4 \mathrm{~mm}$. deep) with a central dimple filled in the usual site of the fossa ovalis (Fig. 1). This membrane was convex towards the right atrium; the central dimple disappeared after fixation.

There was no communication between the atria. Neither right atrium nor right ventricle were distended or hypertrophied. The tricuspid and pulmonary valves were normal. Four pulmonary veins entered a small left atrium which was slightly smaller than the left auricle, itself of normal size. The wall of the atrium was grey-white in colour. The apex of the heart and a third of its left border were formed by the right ventricle, the left ventricular chamber being a small appendage attached to the right; the thickness of the left ventricle was normal $(10 \mathrm{~mm}$.). The thin translucent cusps of a normal mitral valve contrasted sharply with the silvery-grey endocardial lining of the left ventricle (Fig. 2). Tiny nodular thickenings were present on the ventricular aspect of the aortic cusps. The coronary ostia were normal in position and the branches of the coronary arteries were patent. The ascending and transverse parts of the thoracic aorta were of small calibre $(8 \mathrm{~mm}$. circumference) and a further narrowing $(5 \mathrm{~mm}$. circumference and $3 \mathrm{~mm}$. long) occurred immediately proximal to the junction of the aorta with a wide ductus arteriosus $(20 \mathrm{~mm}$. circumference) the internal lining of which was corrugated and of grey-yellow colour. The descending thoracic aorta was of normal circumference $(15 \mathrm{~mm}$.).

The lungs were congested and pieces from all lobes sank in water. No abnormality was found in other organs.

Microscopic Findings. A section of left ventricle showed elastic thickening of the endocardium. Dilated blood channels were seen in the subjacent myocardium; serial sections demonstrated their communication with the cavity of the left ventricle (Fig. 3). A section through the superior part of the obliterated foramen 


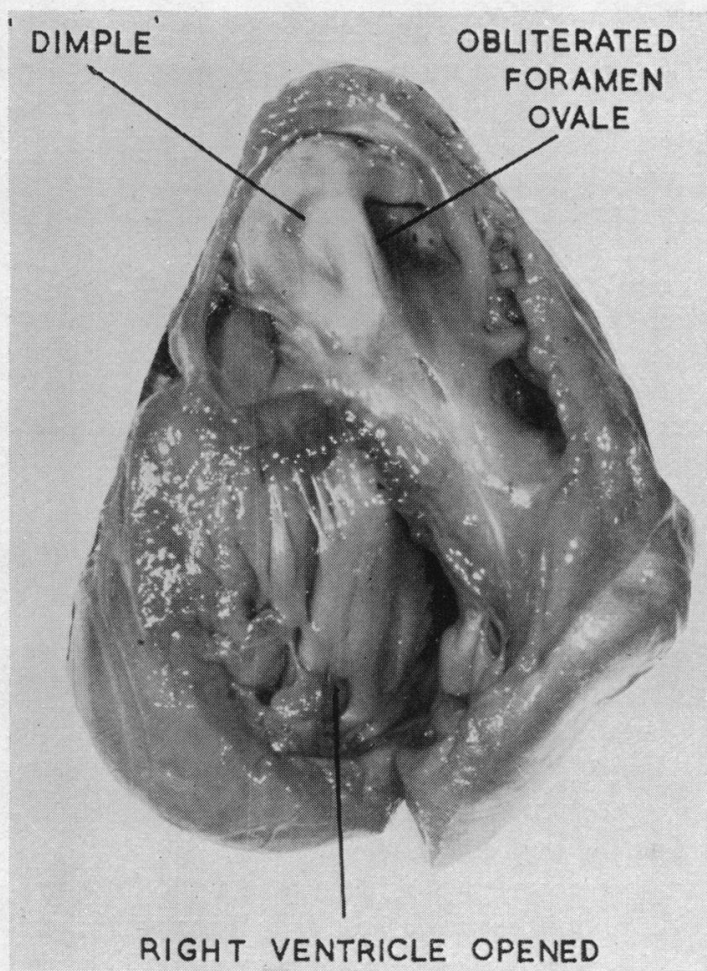

FIG. 1.-Right atrium and ventricle opened before fixation, showing dimple in obliterated foramen ovale.

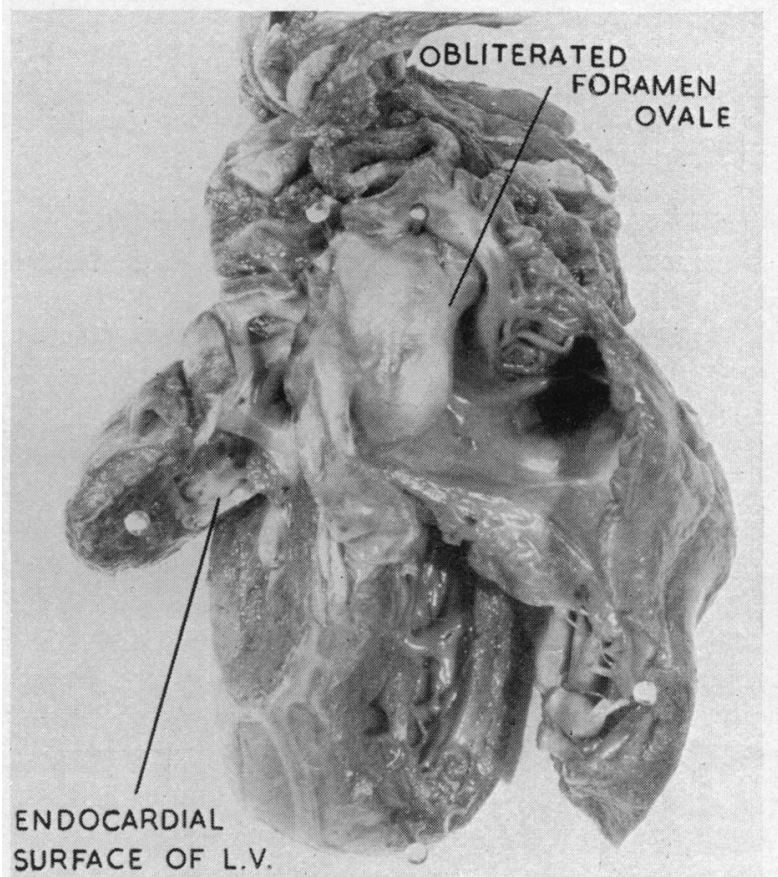

FIG. 2.-Showing the relative proportions of right and left ventricle and the thickened left ventricular endocardium.

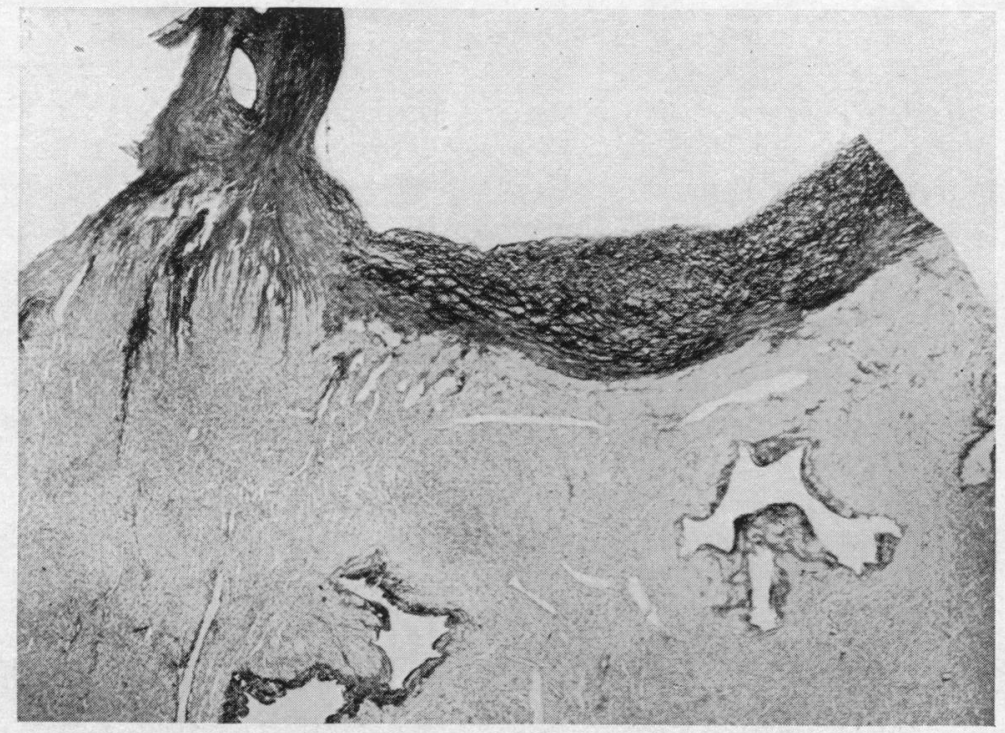

FIG. 3.-Section of left ventricle, showing elastic thickening of the endocardium and dilated subendocardial sinusoids (Weigert's elastic, $\times 28$ ). 
ovale showed normal endocardium on each side and in the superior part of the section a longitudinal slit lined by endothelium beneath which were strands of collagen and elastic tissue. This slit most probably represents a space between the fused septum primum and septum secundum. A thick layer of cardiac muscle, beneath the endocardium, mainly on the left side would explain, by shrinkage, the disappearance of the dimple in the obliterated foramen ovale which was present before fixation.

Compared with controls the left atrial endocardium was not thickened nor was fibroelastosis found in the right atrium or ventricle.

The only other histological abnormalities were seen in the lungs. Sections from all lobes, specifically stained for elastic tissue showed a slight but significant increase of elastic in the intima and media of arterioles as compared with controls. Many macrophages in alveoli contained a black pigment some of which stained blue with Perls' and with Turnbull's methods. Repeated washings of the sections with a saturated solution of picric acid in 85 per cent alcohol failed to remove the remainder of the pigment which was hence unlikely to be derived from formalin.

\section{Discussion}

Only eight reported cases that can be considered to be examples of premature obliteration of the foramen ovale upon the evidence provided have been found.

The first, in a child " of a livid colour" living 36 hours, is that of Vieussens (1715) (quoted by Corvisart, 1813). "The lungs were much swollen and their vessels gorged with blood; the right ventricle and the pulmonary artery were very much dilated; and no trace could be found of the foramen ovale." No further details of the heart are given. An example of this condition in a child, one day old, described by Morgagni (1761) has escaped mention in previous reports. However, as in the last case, information is scanty concerning the rest of the heart.

Smith (1846) contributed a specimen of a heart showing premature obliteration of the foramen ovale to the museum of Guy's Hospital; unfortunately it is no longer available. The heart was taken from a child which was cyanosed within five minutes after the beginning of respiration, deeply cyanosed and comatose in eleven hours, and dead in twenty-one hours. "The fossa ovalis ... entirely closed by a strong reticulated membrane firmly attached to its annulus. and forming a pouch projecting towards the left side. ... The left ventricle was almost obliterated ... lined by a very dense smooth membrane." Bulging of the obliterated foramen ovale towards the left atrium would agree with the expected raised pressure on the right side of the heart. In the present case the obliterated foramen bulged to the right; this may have been due to post-mortem contraction of the thicker mass of cardiac muscle on the left side of the atrial septum compared with that on the right side.

A case is described by Vernon (1856) of absent ductus arteriosus, overriding aorta, small left ventricle, and a ventricular septal defect together with a closed foramen ovale in a child cyanosed at birth and living four hours. However, to say that the foramen ovale is closed, not obliterated, in a newborn child is to state a normal finding and it is doubtful if this case can be included as an example of obliteration of the foramen ovale. Post-mortem examinations of stillborn and neonatal infants reveal in most cases a closed foramen ovale but usually a fine probe can be made to pass from right to left atrium through a postero-superior valvular slit (Fig. 4). This slit can be made visible, in some cases, by altering the position of the heart while looking into the opened right atrium. Johnson (1952) quotes six cases of closed foramen ovale in association with fibroelastosis from a paper by Craig (1949); this latter author states that the foramen ovale was closed but does not mention if a valve was present. It is probable that the foramen was valvular in these cases as the author does not emphasize or comment upon the "closed" foramen ovale. Osler (1880) and Brenner (1939) both regard a valvular foramen ovale at birth as being prematurely closed: the detailed studies of Patten (1938) show that such a concept is not now tenable. The case described by Osler and that of Tait (1875) were probably examples of hydrops fotalis though insufficient detail is provided to be certain. Generalized œdema at birth was a feature of both of these cases and Lehman (1927) considered that this formed one of the clinical variants of premature closure of the foramen ovale; the other two varieties being cases with progressive cyanosis leading to death 
and those in which the abnormality was associated with multiple cardiac defects. There are no grounds for including those cases with valvular foramen ovale showing generalized œdema at birth as examples of obliteration of the foramen ovale.

The clinical picture in all reported cases of obliteration of the foramen ovale, without septal defects or overriding great vessels, was progressive cyanosis leading to death, and in these cases the left ventricle showed fibroelastosis as judged by microscopic appearances in all except that of Smith

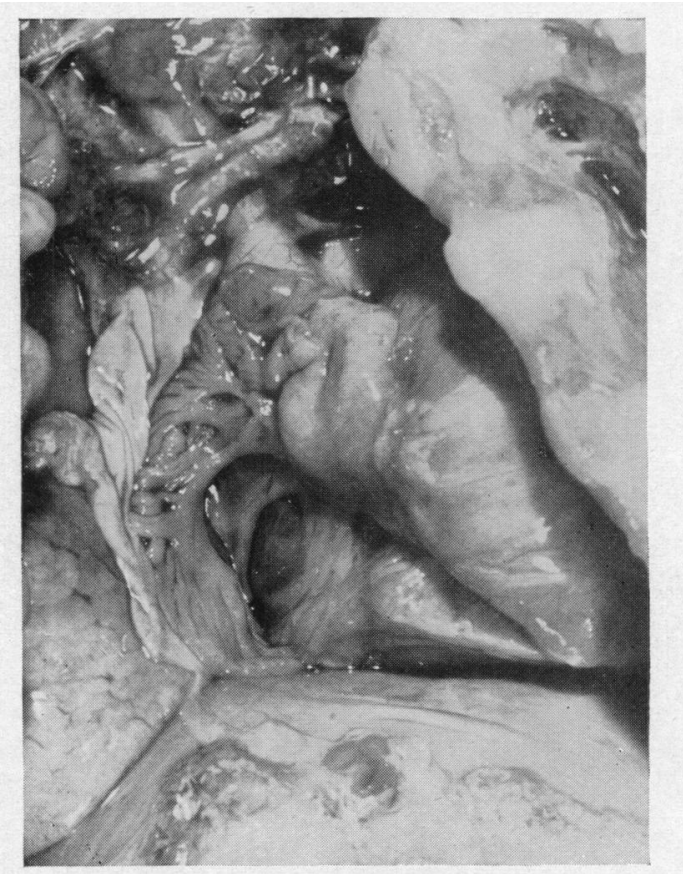

FIG. 4.-Heart of a newborn child (aet. 10 hours); right atrium opened in situ to show closed foramen ovale.

(1846); in this case the macroscopic appearances suggested fibroelastosis. The constant presence of ventricular fibroelastosis in those cases in which adequately oxygenated blood is practically excluded from the left ventricle supports the view of Johnson (1952) that anoxia of the ventricular endocardium plays a part in the production of fibroelastosis. This concept is strengthened by the fact that the coronary circulation is developed progressively throughout embryonic life; the myocardial blood supply being first derived from sinusoids communicating with the lumen of the ventricle. It is probable that obliteration of the foramen ovale early in intrauterine life would lead to severe endocardial anoxia which may be responsible for the fibroelastic thickening of the ventricle. The small size of the atrial, ventricular, and aortic cavities may be explained by the diminished blood flow through them. In the present case the aorta was hypoplastic in its ascending and part of its transverse portion and showed an area of coarctation proximal to its junction with the ductus arteriosus. A more severe degree of aortic narrowing producing atresia, in a case with obliteration of the foramen ovale and atresia of the mitral orifice, is reported by Bellet and Gouley (1932): here also the left ventricle was small and showed fibroelastosis. A curious observation is the normal and sometimes increased thickness of the wall of the left ventricle in these cases despite the diminished flow through the chamber, in utero.

The degree of fibroelastic involvement of the left atrium is difficult to assess as the amount of subendocardial elastic and collagen varies throughout the atrial endocardium. In control cases the 
amount of elastic is greatest in the posterior parts of the left atrium and hence the site from which a section is taken must be clearly stated before an opinion is expressed as to the degree of elastic thickening of the endocardium. Several authors (Johnson, 1952; Bellet and Gouley, 1932; and Edwards and Du Shane, 1950) state that fibroelastosis was present in the left atria in their cases. In the present case the macroscopic appearances of the atrium were suggestive of fibroelastosis but comparison of sections with controls showed very little difference in the amount of elastic present.

The membrane obliterating the foramen ovale in the case here reported consisted almost entirely of muscle. This suggests that the obliteration was due to abnormal development rather than to inflammatory thickening; the former view is supported by Patten (1938) who postulates failure of development of the septum secundum as the cause. Against this view is the slit found in the present case in sections through the obliterated foramen ovale that may represent a space between septum primum and septum secundum. A few strands of muscle are found in sections through the foramen ovale of the newborn; however, they undoubtedly undergo hypertrophy in the obliterated foramen ovale as seen in the present case due to the increased pressure in the right atrium.

The course of the circulation in utero in this infant was probably abnormal from about the eighth week - the time of completion of development of the atrial septum. Most of the blood received by the right atrium would probably pass through the ductus arteriosus to the aorta. Despite this the pulmonary blood flow was probably greater than is normal in fœtal life and this may account for the slight degree of intimal hypertrophy seen in the pulmonary blood vessels. The source of the alveolar pigment is obscure.

The progressive cyanosis at birth may be explained by shrinkage of the ductus producing an increased blood flow through the small left side of the heart; not only was the volume of blood too great for the small chambers but also the aortic resistance would be high and this no doubt led to acute cardiac failure.

\section{SUMMARY}

The ninth case of premature obliteration of the foramen ovale is described. Previously reported cases are reviewed and discussed.

The constant finding of left ventricular fibroelastosis in these cases supports the view that anoxia plays a part in its production.

The valvular nature of the foramen ovale at birth is emphasized.

I am grateful to Dr. D. Gairdner for permission to publish this case, to Dr. A. M. Barrett for his constant encouragement and advice, to Dr. B. Towers for his patience in explaining the embryological details, and to Dr. Keith Simpson for information concerning the specimen described by Smith (1846). I wish to thank Miss S. Westoby and Mr. S. Patman for the photographs.

\section{REFERENCES}

Bellet, S., and Gouley, B. A. (1932). Amer. J. med. Sci., 183, 458.

Brenner, M. C. (1939). Amer. Heart J., 17, 437.

Corvisart, J. N. (1813). A Treatise on the Diseases and Organic Lesions of the Heart and Great Vessels. (Translated by C. H. Hebb.) Underwood and Blacks, London.

Craig, J. M. (1949). Bull. Internat. Ass. med. Mus., 30, 15.

Edwards, J. E., and Du Shane, J. W. (1950). Arch. Path., 49, 517.

Johnson, F. R. (1952). Arch. Path., 54, 237.

Lehman, E. (1927). Amer. J. Dis. Child., 33, 585.

Morgagni, J. B. (1761). De Sedibus et Causis Morborum. Lib. III, Epist. 48.

Osler, W. (1880). Montreal Gen. Hosp. Rep., 1, 177.

Patten, B. M. (1938). Amer. J. Path., 14, 135.

Smith, E. (1846). Tr. Path. Soc., London, 1, 52.

Tait, L. (1875). Tr. Obst. Soc., London, 17, 307.

Vernon, H. H. (1856). Tr. M. Chir., London, 39, 297. 